\section{Wie gut ist die minimalinvasive Magenchirurgie?}

In einer Metaanalyse wurde der Nutzen der laparoskopisch-assistierten distalen Gastrektomie mit D2-Dissektion im Vergleich zur offenen Operation bei der Behandlung von Magenkrebs untersucht.

$\mathrm{B}$ ei der Behandlung von Magenkarzinomen werden die Wahl des Operationsverfahrens, der Umfang der Resektion und der Level der Radikalität noch immer kontrovers diskutiert. Eine laparoskopisch-assistierte distale Gastrektomie mit D2-Dissektion ist technisch machbar, aber bei größeren Dissektionen fehlt die Bestätigung, dass sie noch den

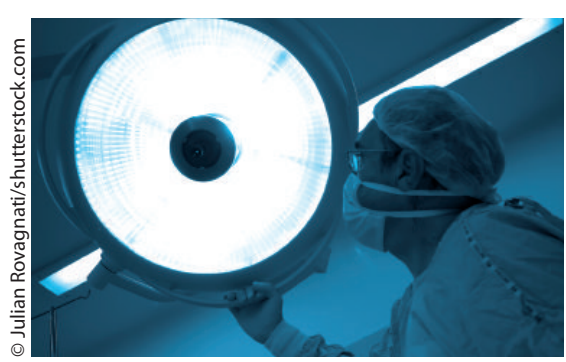

Genau beleuchtet: Vor- und Nachteile der laparoskopisch-assistierten distalen Gastrektomie.
Vorteil minimaler Invasivität in Anspruch nehmen kann. Außerdem ist unklar, ob perioperative Komplikationen und die Mortalität zunehmen und ob dasselbe Ausmaß an Radikalität erreicht werden kann wie bei einer offenen Operation.

Um den Wert der laparoskopisch-assistierten distalen Gastrektomie mit D2Dissektion beurteilen zu können, unternahm die chinesische Arbeitsgruppe um Jie Ding eine Metaanalyse von acht retrospektiven Fall-Kontroll-Studien aus den vergangenen 15 Jahren mit insgesamt 1.065 Patienten, in denen die minimal invasive Methode mit der offenen Operation verglichen worden war.

Die laparoskopische Gastrektomie dauerte signifikant länger als die offene. Dafür war der Blutverlust signifikant niedriger, und es traten um 39\% weniger Komplikationen auf. Außerdem waren beim minimal invasiven Vorgehen die
Zeit bis zum ersten Flatus als Kriterium für das Wiedererlangen der MagenDarm-Funktion, die Zeit bis zur ersten Nahrungsaufnahme und die Dauer des Klinikaufenthalts signifikant kürzer. Keine signifikanten Unterschiede gab es bei der Zahl der abgetragenen Lymphknoten, der Mortalität und den Rezidivraten.

Anzumerken ist, dass lediglich retrospektive Studien analysiert wurden, die gewissen Limitationen unterliegen. Für eindeutige Ergebnisse wären prospektive randomisierte Studien wünschenswert.

Fazit: Die Ergebnisse dieser Metaanalyse bestätigen einige Vorteile der laparoskopisch-assistierten distalen Gastrektomie mit D2-Dissektion gegenüber der offenen Operation, z. B. eine schnellere Genesung und eine geringere Komplikationsrate. Nachteile sind die etwas längere Operationsdauer und die unklare Langzeitprognose.

Judith Neumaier

Ding J et al. Meta-analysis of laparoscopyassisted distal gastrectomy with D2 lymph node dissection for gastric cancer. J Surg Oncol. 2012;105(3):297-303.

\section{Familienanamnese hilft bei Prognose}

Lässt sich die postoperative Prognose von Patienten mit Magenkrebs im Stadium III-IV anhand der Familienanamnese beurteilen? Koreanische Onkologen untersuchten das in einer retrospektiven Studie mit über 1.200 Patienten.

D as persönliche Risiko, an Magenkrebs zu erkranken, ist etwa 3,7-mal größer, wenn ein Familienmitglied ersten Grades - Eltern, Kinder, Geschwister - bereits am gleichen Tumor erkrankt ist. Südkoreanische Onkologen prüften jetzt in einer großen retrospektiven Studie, ob eine solche Familienanamnese mit einer besseren Prognose bei solchen Patienten assoziiert ist, die wegen eines Adenokarzinoms des Magens mit kurativer Intention operiert worden waren. Für die Studie wurden Daten von 1.273 Patienten ausgewertet, von denen 263 Familienangehörige ersten Grades mit einem Magenkarzinom hatten.

Ähnlich wie bei Patienten mit Brustoder Darmkrebs waren fünf Jahre nach Studienbeginn in der koreanischen Studie Gesamt (OS)-, rezidivfreies (RFS) und krankheitsfreies Überleben (DFS) deutlich höher bei Patienten mit einem an Magenkrebs erkrankten Angehörigen. Vergleichsgruppe waren Magenkrebs-Patienten ohne positive Familienanamnese.

Signifikante Unterschiede bei diesen Parametern im Vergleich zu Patienten ohne entsprechende Familienanamnese gab es allerdings nur bei Patienten in den fortgeschrittenen Erkrankungsstadien III und IV. Hier lagen die adjustierten HR (Hazard Ratio)-Werte für den Parameter OS bei 0,47, für DFS bei 0,49 und für RFS bei 0,51 . Herausgerechnet wurden unter anderem die Parameter Alter, Rauchen, Tumorgröße und -lokalisation sowie Lymphknotenmetastasen.

Auch wenn die positive Assoziation bestätigt wurde, ist noch immer unklar, wie eine positive Familienanamnese die Prognose verbessert. Eine Erklärung könnte den koreanischen Onkologen zufolge sein, dass Magenkrebs-Patienten, die ebenfalls daran erkrankte Verwandte ersten Grades haben, sich mehr als andere um eine gesunde Lebensweise bemühen.

Eine andere Erklärung könnte ihrer Ansicht nach die Mikrosatelliteninstabilität (MSI) sein, das sind Längenveränderungen in der DNA, die bei etwa einem Drittel der Magenkrebs-Patienten vorkommen und mit einer verbesserten $\mathrm{Ge}$ samtprognose assoziiert sind. MSI hat wie bei Patienten mit einem Kolonkarzinom offenbar einen schützenden Effekt.

Fazit: Wenn Patienten mit einem Magenkarzinom Eltern, Geschwister oder Kinder mit der gleichen Tumorerkrankung haben, könnte das die postoperative Prognose bei Patienten im Stadium III oder IV verbessern.

Peter Leiner

Han MA et al. Association of family history with cancer recurrence and survival in patients with gastric cancer. J Clin Oncol. 2012; 30(7):701-8. 